\title{
Stochastic bifurcation in noise-driven lasers and Hopf oscillators
}

\author{
Sebastian Wieczorek \\ Mathematics Research Institute, University of Exeter, EX4 4QF, United Kingdom \\ (Received 8 October 2008; revised manuscript received 23 January 2009; published 24 March 2009)
}

\begin{abstract}
This paper considers nonlinear dynamics in an ensemble of uncoupled lasers, each being a limit-cycle oscillator, which are driven by the same external white Gaussian noise. As the external-noise strength increases, there is an onset of synchronization and then subsequent loss of synchrony. Local analysis of the laser equations shows that synchronization becomes unstable via stochastic bifurcation to chaos, defined as a passing of the largest Lyapunov exponent through zero. The locus of this bifurcation is calculated in the threedimensional parameter space defined by the Hopf parameter, amount of amplitude-phase coupling, and external-noise strength. Numerical comparison between the laser system and the normal form of Hopf bifurcation uncovers a square-root law for this stochastic bifurcation as well as strong enhancement in noiseinduced chaos due to the laser's relaxation oscillation.
\end{abstract}

DOI: 10.1103/PhysRevE.79.036209

PACS number(s): 05.45.Xt, 42.60.Mi

\section{INTRODUCTION}

Synchronization of nonlinear oscillators to external noise is an interesting mathematical problem of importance in biology, applied science, and engineering [1-9]. The key difference to synchronization to an external periodic signal is the lack of a simple functional relationship between the noisy input and the synchronized output, making the phenomenon much less evident $[10,11]$. Rather, synchronization to noise is defined as identical response of two or more identical but uncoupled oscillators to the same external noise. This definition is equivalent to obtaining reproducible output from a single oscillator driven repeatedly by the same external noise, each time starting at a different initial state. Hence, noise synchronization is also known as reliability [2] or consistency [6], and represents the ability to encode irregular external signals in a reproducible manner.

Recent studies have shown that limit-cycle oscillators can exhibit interesting nonlinear response to noisy external signals. Typically, a small amount of external noise causes synchronization which is quantified by negative largest Lyapunov exponent of the noise-driven system [1,3]. However, as the external-noise strength increases, one can observe loss of synchrony in oscillators with amplitude-phase coupling (also called nonisochronicity or shear) [7-9,12,13]. The resulting asynchronous behavior is quantified by positive largest Lyapunov exponent of the noise-driven system. Mathematically, loss of synchrony, consistency, or reliability is a manifestation of the same phenomenon, namely, stochastic bifurcation defined as a crossing of the largest Lyapunov exponent through zero. This paper focuses on such stochastic bifurcation in lasers and Hopf oscillators, and contributes to the general problem of noise-induced nonlinear dynamics.

Single-mode lasers are especially interesting as they are experimentally accessible amplitude and phase oscillators. The amplitude-phase coupling is quantified by the linewidth enhancement factor that varies with the laser type and design $[14,15]$. Externally driven lasers have been intensively studied for fundamental understanding of synchronization properties and for technological applications such as high-power light generation from phased laser arrays [16-18]. Synchro- nization and instabilities in lasers with periodic external signal [19] are well understood; see [20] for an overview of the literature. However, only a few works considered nonlinear dynamics in lasers with external noise [6,21]. In particular, no stochastic bifurcation has yet been reported in whitenoise-driven lasers.

Motivated by fundamental interest and applications alike, we address whether external white noise can induce phase coherence or interesting dynamics in an ensemble of uncoupled lasers with intrinsic noise. We demonstrate onset of noise synchronization similar to that observed when the external signal is periodic. Then, one transition to asynchronous behavior and another transition back to synchrony is found for nonzero linewidth enhancement factor. Each transition corresponds to stochastic bifurcation in a single laser with external white noise. We study this bifurcation with dependence on the three parameters: the laser pump which controls the distance from Hopf bifurcation of the noise-free laser, the linewidth enhancement factor which quantifies the amount of amplitude-phase coupling, and the external-noise strength. The analysis uncovers an experimentally accessible region of chaos (sensitivity on initial conditions) that is purely noise induced; the noise-free laser does not possess any type of a chaotic set in its phase space. For small external-noise strength, the numerically obtained stochastic bifurcation in the laser system follows a square-root law in agreement with the normal form of Hopf bifurcation. However, with increasing external-noise strength, discrepancies arise. In the laser system, the noise-induced chaos is significantly enhanced due to one additional degree of freedom and damped relaxation oscillation toward the laser's limit cycle.

\section{LASER RATE EQUATIONS}

The analysis is based on the stochastic rate equations for a single-mode semiconductor laser with intrinsic spontaneous emission noise. Time evolution of $M$ uncoupled lasers injected with common external optical signal $f_{\text {ext }}(t)$ can be described as $[19,21]$

$$
\frac{d E_{j}}{d t}=i \Delta E_{j}+\beta \gamma(1-i \alpha) N_{j} E_{j}+f_{E j}(t)+f_{e x t}(t),
$$




$$
\begin{gathered}
\frac{d N_{j}}{d t}=\Lambda-N_{j}-\left(1+\beta N_{j}\right)\left|E_{j}\right|^{2}+f_{N j}(t), \\
j=1,2, \ldots, M,
\end{gathered}
$$

where, $E_{j}=E_{j}^{R}+i E_{j}^{I}$ is the normalized complex electric field and $N_{j}$ is the normalized deviation from the threshold density of excited carriers (electron-hole pairs) in laser $j . \Lambda$ is the normalized deviation from the threshold pump rate. $\left(N_{j}\right.$ $=-1$ corresponds to zero carrier density and $\Lambda=-1$ corresponds to zero pump rate.) The linewidth enhancement factor $\alpha$ quantifies amplitude-phase coupling of the complex field, $\Delta$ is the normalized detuning between the free-running laser frequency and some conveniently chosen reference frequency, $\gamma=500$ is the normalized decay rate, and $\beta=2.765$ is the normalized gain coefficient $[21,23]$. (Different values of $\beta$ and $\gamma$ are used in Fig. 7 as indicated in the caption.) The lasers are identical except for the spontaneous emission noise represented by random Gaussian processes $f_{E j}(t)=f_{E j}^{R}(t)$ $+i f_{E j}^{I}(t)$ and $f_{N j}(t)$ which have zero mean and are $\delta$ correlated

$$
\begin{gathered}
\left\langle f_{E j}(t)\right\rangle=\left\langle f_{N j}(t)\right\rangle=0, \\
\left\langle f_{E i}^{R}(t) f_{E i}^{I}(t)\right\rangle=0, \\
\left\langle f_{E i}^{R}(t) f_{E j}^{R}\left(t^{\prime}\right)\right\rangle=\left\langle f_{E i}^{I}(t) f_{E j}^{I}\left(t^{\prime}\right)\right\rangle=D_{E} \delta_{i j} \delta\left(t-t^{\prime}\right), \\
\left\langle f_{N i}(t) f_{N j}\left(t^{\prime}\right)\right\rangle=2 D_{N} \delta_{i j} \delta\left(t-t^{\prime}\right) .
\end{gathered}
$$

Here, $\delta_{i j}$ is the Kronecker $\delta$ and $\delta\left(t-t^{\prime}\right)$ is the Dirac $\delta$ function. In the calculations we use $2 D_{E}=0.1$ and $2 D_{N}$ $=6.9 \times 10^{-8}[23]$.

A single laser without noise can be described using Eqs. (1) and (2) with $f_{E j}(t)=f_{\text {ext }}(t)=f_{N j}(t)=0$ which define a threedimensional dynamical system. This system is $\mathrm{S}^{1}$ equivariant and has rotational symmetry corresponding to a phase shift $E \rightarrow E e^{i \phi}$, where $0<\phi \leqslant 2 \pi$. For relevant values of $\Lambda>-1$ there is an equilibrium at $\left(E_{e q}, N_{e q}\right)=(0, \Lambda)$ which represents the "off" state of the laser. This equilibrium is globally stable if $-1<\Lambda<0$ and unstable if $\Lambda>0$. At $\Lambda=0$, there is a Hopf $(\Delta \neq 0)$ or pitchfork $(\Delta=0)$ bifurcation which defines the laser threshold. Moreover, if $\Lambda>0$, the system has a stable group orbit in the form of periodic orbit $(\Delta \neq 0)$ or the whole circle of equilibria $(\Delta=0)$. In the paper, we refer to this circular attractor as the limit cycle. The limit cycle is given by $\left(\left|E_{0}\right|^{2}, N_{0}\right)=(\Lambda, 0)$ and represents the "on" state of the laser.

The amplitude-phase coupling $\alpha$ of the complex field $E$ is crucial to our analysis. Its physical origin is the dependence of the semiconductor refractive index (and hence the cavity resonant frequency) on the number of carriers $[14,20]$. A change in the electric field intensity $|E|^{2}$ induces a change $\delta N$ in the number of carriers [Eq. (2)]. The resulting change in the refractive index shifts the cavity resonant frequency by $-\alpha \beta \gamma \delta N$ [Eq. (1)]. The ultimate result is a change in the (instantaneous) frequency of the electric field $E$. Mathematically, amplitude-phase coupling is best illustrated by a special set associated with a point $p$ on the limit cycle. This set is defined as a stable manifold of $p(t)$,
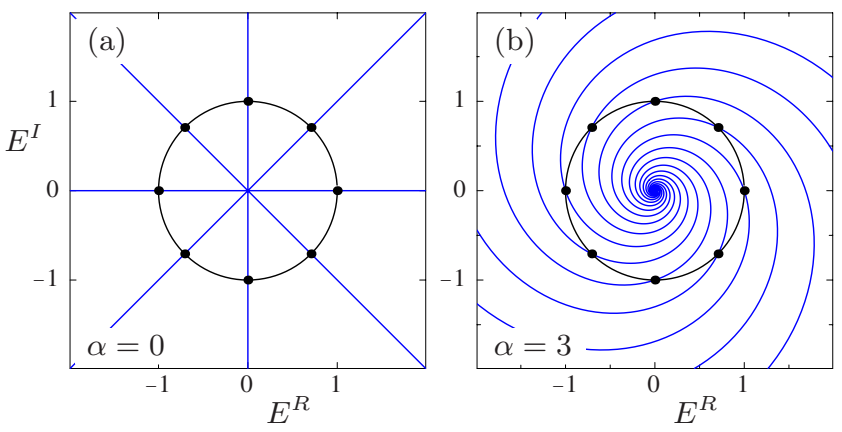

FIG. 1. (Color online) Projection onto the complex $E$ plane of (black) the limit cycle representing the "on" state of the laser and (blue) isochrones of (black dots) eight different points on the limit cycle as defined by Eq. (4). Each two-dimensional isochron is independent of $N$ and, hence, appears as a one-dimensional curve in the projection. $\Lambda=1$ and (a) $\alpha=0$ and (b) $\alpha=3$.

$$
W^{s}(p(t))=\{x: x(t) \rightarrow p(t) ; t \rightarrow \infty\},
$$

and is called an isochron [22]. Isochrones of eight different points on the laser limit cycle are shown in Fig. 1 in projection onto the complex $E$ plane. Isochron inclination to the orthogonal to the limit cycle at $p$ indicates the strength of phase-space stretching along the limit cycle. If $\alpha=0$, all trajectories with different initial $|E|>0$ rotate around the origin of the $E$ plane with the same average frequency giving no isochron inclination nor phase-space stretching [Fig. 1(a)]. However, if $|\alpha|>0$, trajectories with larger initial $|E|$ rotate with higher average frequency [Fig. 1(b)] giving rise to isochron inclination and phase-space stretching [Fig. 1(b)]. The amplitude-phase coupling (or nonisochronicity) plays an important role in noise synchronization [9] and is responsible for the interesting nonlinear response reported in Secs. III and IV of this paper.

\section{SYNCHRONIZATION BY EXTERNAL SIGNALS}

Phased multilaser systems have been studied over decades for fundamental interest and potential applications, for example, in generation of high intensity light $[17,18]$. If one focuses light from $M$ identical lasers onto a small spot of the order of a wavelength, the total average intensity at this spot is given by

$$
\left\langle I_{M}(t)\right\rangle=\lim _{T \rightarrow \infty} \frac{1}{T} \int_{0}^{T}\left|\sum_{j=1}^{M} E_{j}(t)\right|^{2} d t .
$$

A single laser oscillates with random phase owing to spontaneous emission noise $f_{E j}(t)$ [Eq. (3)]. When the lasers are independent, the total average intensity at the focal spot is proportional to $M$ times the average intensity of a single laser. This follows directly from Eq. (5) assuming lasers with identical amplitudes and uncorrelated random phases. However, when the lasers oscillate in phase, one expects the total average intensity at the focal spot of $M^{2}$ times the average intensity of a single laser. This follows directly from Eq. (5) assuming lasers with identical amplitudes and phases. We will now consider the synchronizing effect of two different 


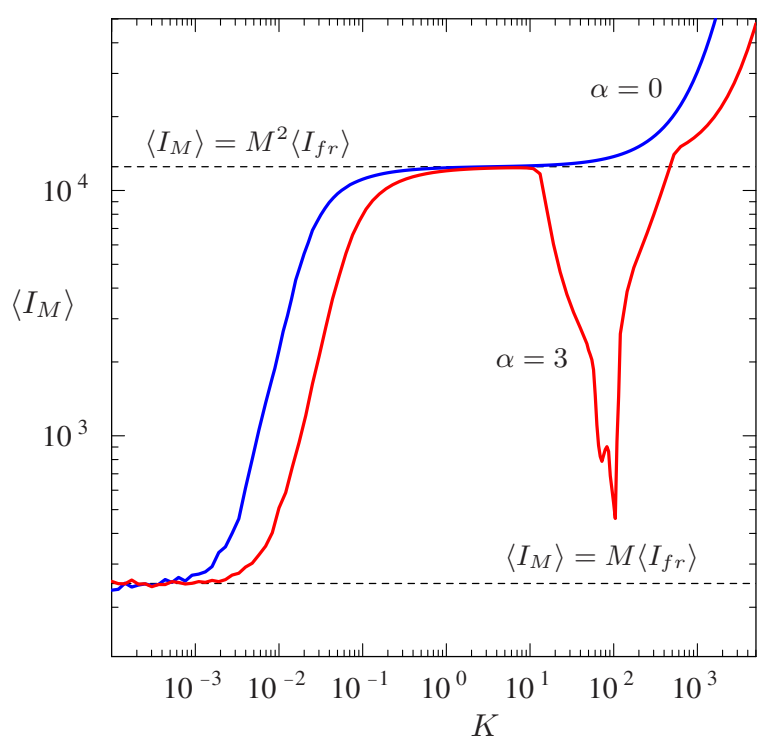

FIG. 2. (Color online) Total average intensity as defined by Eq. (5) of $M=50$ independent lasers with common coherent external signal vs the external-signal amplitude $K$ for (blue) $\alpha=0$ and (red) $\alpha=3 ; \Lambda=5$ and $\nu_{\text {ext }}=\Delta$.

external signals $f_{\text {ext }}(t)$ and use the total average intensity as given by Eq. (5) to measure the quality of synchronization. For comparability reasons, we briefly review the case of a pure-coherent external signal and then move on to the case of a pure-incoherent (white noise) external signal.

A standard approach to obtaining phased laser arrays uses a weak coherent external signal

$$
f_{\text {ext }}(t)=K e^{i \nu_{\text {ext }} t},
$$

where $K \in \mathbb{R}$ is the external-signal amplitude and $\nu_{\text {ext }}$ is the detuning between the external-signal frequency and the chosen reference frequency. Such an external signal breaks $\mathrm{S}^{1}$ symmetry and can force each laser to fluctuate in the vicinity of the well-defined external signal's phase $\nu_{\text {ext }} t$ [24], as opposed to a random walk. Figure 2 shows $\left\langle I_{M}(t)\right\rangle$ versus the amplitude $K$ of the external signal resonant with the lasers $\left(\nu_{\text {ext }}=\Delta\right)$. Because of the intrinsic spontaneous emission noise, the external-signal strength has to reach a certain threshold before synchronization occurs. For $\alpha=0$, a sharp onset of synchronization at $K \approx 10^{-3}$ is followed by a wide range of synchronous behavior with $\left\langle I_{M}(t)\right\rangle=M^{2}\left\langle I_{f r}(t)\right\rangle$, where $\left\langle I_{f r}(t)\right\rangle$ is the average intensity of a free-running (no external signal) laser. At around $K=10^{2},\left\langle I_{M}(t)\right\rangle$ starts increasing above $M^{2}\left\langle I_{f r}(t)\right\rangle$. Whereas lasers still remain synchronized, this increase indicates that the external signal is no longer small. Rather, it starts dominating the dynamics and up shifts the average intensity of each individual laser. A different scenario is observed for $\alpha=3$. There, the onset of synchronization is followed by an almost complete loss of synchrony just before $\left\langle I_{M}(t)\right\rangle$ increases above $M^{2}\left\langle I_{f r}(t)\right\rangle$. The loss of synchrony is caused by externally induced bifurcations and ensuing chaotic dynamics. These bifurcations have been studied in detail, both theoretically [20,25-27] and experimentally [28], and are well understood.

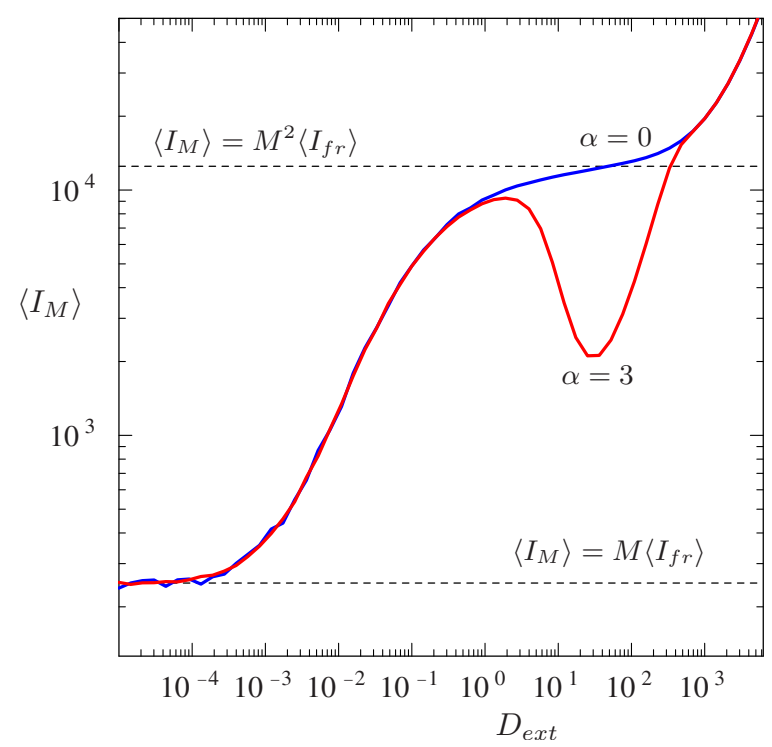

FIG. 3. (Color online) Total average intensity as defined by Eq. (5) of $M=50$ independent lasers with common external white noise vs the external-noise variance $D_{\text {ext }}$ for (blue) $\alpha=0$ and (red) $\alpha=3$; $\Lambda=5$.

Here, we study an alternative approach that uses a pureincoherent external signal represented by the complex random process that is Gaussian, has zero mean, and is $\delta$ correlated,

$$
\begin{gathered}
f_{\text {ext }}(t)=f_{\text {ext }}^{R}(t)+i f_{\text {ext }}^{I}(t), \\
\left\langle f_{\text {ext }}(t)\right\rangle=\left\langle f_{\text {ext }}^{R}(t) f_{\text {ext }}^{I}(t)\right\rangle=0, \\
\left\langle f_{\text {ext }}^{R}(t) f_{\text {ext }}^{R}\left(t^{\prime}\right)\right\rangle=\left\langle f_{\text {ext }}^{I}(t) f_{\text {ext }}^{I}\left(t^{\prime}\right)\right\rangle=D_{\text {ext }} \delta\left(t-t^{\prime}\right) .
\end{gathered}
$$

Such external signal can synchronize the ensemble by forcing each laser to have (nearly) identical random fluctuations. This phenomenon is demonstrated in Fig. 3 where we plot $\left\langle I_{M}(t)\right\rangle$ versus $D_{\text {ext }}$. For $\alpha=0$, one finds a clear onset of synchronization at around $D_{e x t}=10^{-3}$, followed by synchronous behavior at larger $D_{\text {ext }}$. In particular, there exists a range of $D_{\text {ext }}$ where external-noise is strong enough to synchronize phases of intrinsically noisy lasers but weak enough so that each individual laser has small intensity fluctuations and unchanged average intensity $\left\langle I_{f r}(t)\right\rangle$. The resulting probability distributions for the total average intensity $I_{M}(t)$ are shown in blue in Figs. 4(a) and 4(b). The distinct peak at $I_{M}(t)$ $\approx M^{2}\left\langle I_{f r}(t)\right\rangle$ and a noticeable tail at smaller $I_{M}(t)$ indicate synchronization that is not perfect as synchronous behavior is occasionally interrupted with short intervals of asynchrony. For $D_{\text {ext }} \gtrsim 10^{2}$, we observe a trivial case of noiseinduced synchrony where the external noise starts dominating laser dynamics: the intensity of each individual laser exhibits large fluctuations and its average increases above $\left\langle I_{f r}(t)\right\rangle$. As a consequence, the total average intensity $\left\langle I_{M}(t)\right\rangle$ increases above $M^{2}\left\langle I_{f r}(t)\right\rangle$ [Fig. 3] and $I_{M}(t)$ exhibits large fluctuations [Fig. 4(c)] as in the asynchronous case (though the lasers remain in synchrony). A different scenario is again observed for $\alpha=3$. There, the onset of synchronization is 


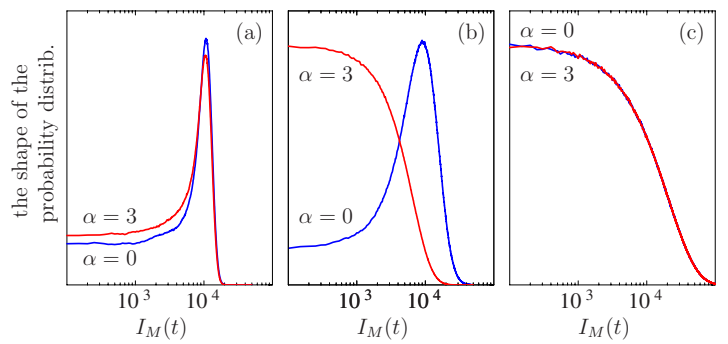

FIG. 4. (Color online) The shape of the probability distribution of $I_{M}(t)$ from Fig. 3 for (blue) $\alpha=0$ and (red) $\alpha=3$. From (a) to (c) $D_{\text {ext }}=10^{0}, 10^{1}$, and $10^{3}$.

followed by a significant loss of synchrony for $4 \lessgtr D_{\text {ext }}$ $\leq 2 \times 10^{2}$. In this parameter interval, one finds qualitatively different dynamics in an ensemble of lasers with $\alpha=0$ and $\alpha=3$ as is further revealed by different probability distributions in Fig. 4(b).

Interestingly, comparison between Figs. 2 and 3 shows that some general aspects of synchronization to a purecoherent and pure-incoherent external signal are similar. In both cases there is a clear onset of synchronization followed by a significant loss of synchrony for nonzero $\alpha$ and subsequent revival of synchronous dynamics under strong external signal. However, the dynamical mechanism responsible for the loss of synchrony in the case of a pure-incoherent external signal (red curve in Fig. 4) has not been studied in laser systems to date and is not fully understood in general.

\section{STOCHASTIC BIFURCATION}

The previous section motivates further research needed to reveal the dynamical mechanism responsible for the loss of synchrony observed in Fig. 4. Therefore, it is useful to first recall some facts about random dynamical systems. In a deterministic (noise-free) laser, lasing is represented by the limit cycle with one zero and two negative Lyapunov exponents (LEs). In the presence of noise, the zero LE shifts to either positive or negative values [9]. In particular, one speaks of a random sink when the largest LE is negative and of a random strange attractor when the largest LE is positive [29]. Note that random attractors are defined only relative to a set of trajectories which are attracted. As such, they provide information about the relative long term behavior of nearby trajectories, as in the deterministic case, but not about the behavior of a single trajectory relative to the origin of the phase space. Qualitative changes of random attractors are defined as crossing of the largest LE through zero and are called stochastic $d$ bifurcations [29]. This is in contrast to stochastic $p$ bifurcations defined as qualitative changes in the phase-space probability distribution that are not necessarily accompanied by crossing of the largest LE through zero.

\section{A. Laser system}

To facilitate the analysis, we henceforth consider $M$ identical lasers that have no intrinsic spontaneous emission noise but are subjected to common external noise. In the absence
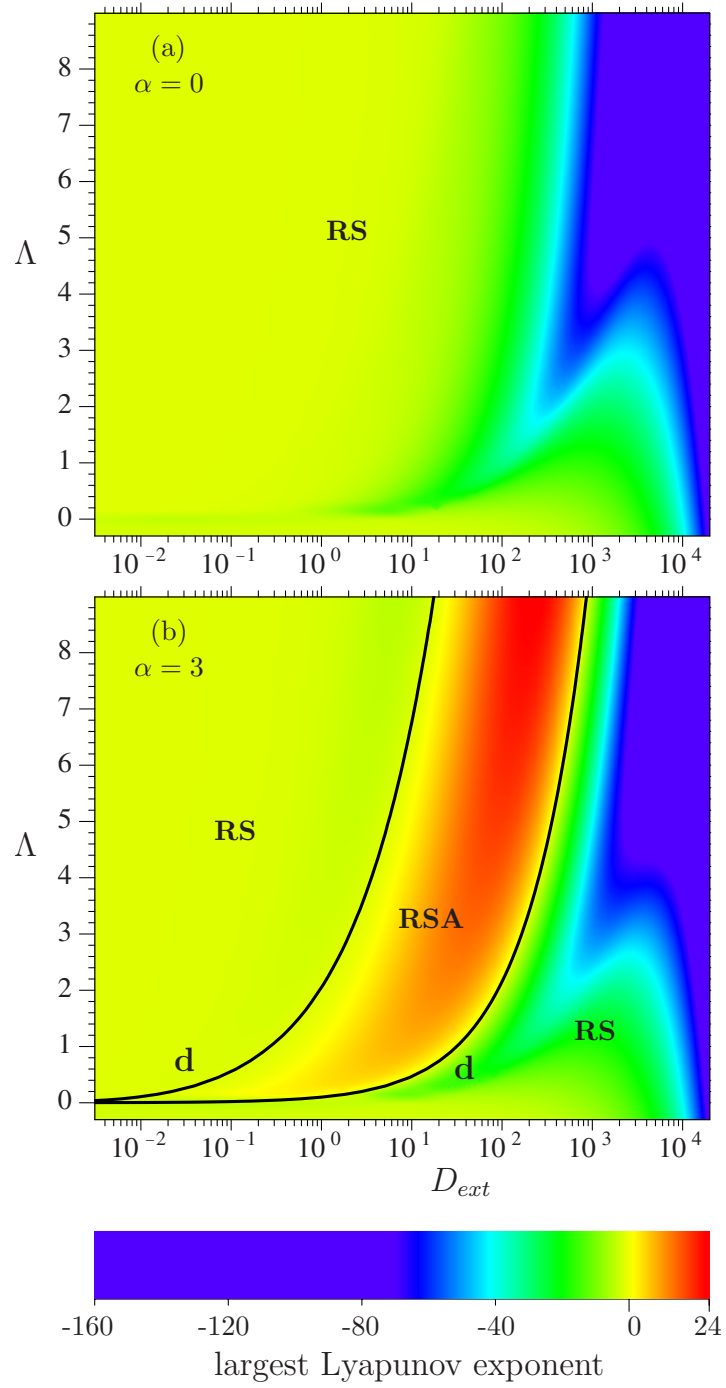

FIG. 5. (Color online) Bifurcation diagram of a single laser with just external noise in the $\left(D_{\text {ext }}, \Lambda\right)$ plane for (a) $\alpha=0$ and (b) $\alpha=3$. RS stands for random sink, RSA stands for random strange attractor, the two black curves denote stochastic $d$ bifurcation, and color coding is for the largest Lyapunov exponent. A noise-free laser has Hopf bifurcation at $\left(D_{\text {ext }}=0, \Lambda=0\right)$.

of the intrinsic noise, one can show that there exists an exact synchronous solution

$$
E_{1}(t)=E_{2}(t)=\cdots=E_{M}(t)=E(t),
$$

whose stability is determined from the sign of the largest LE of just a single laser with external noise. Intuitively, $M$ different trajectories of a single laser with external noise correspond to the evolution of an ensemble of $M$ uncoupled lasers with common external noise. Since LEs measure the exponential rate of separation between nearby trajectories [30], a stable (unstable) synchronous solution is represented by a random sink (a random strange attractor) in a single laser with external noise.

Figure 5 shows effects of external noise on the sign of the otherwise zero largest LE in a single laser. For $\alpha=0$, external noise always shifts the largest LE to negative values so the 
(a)
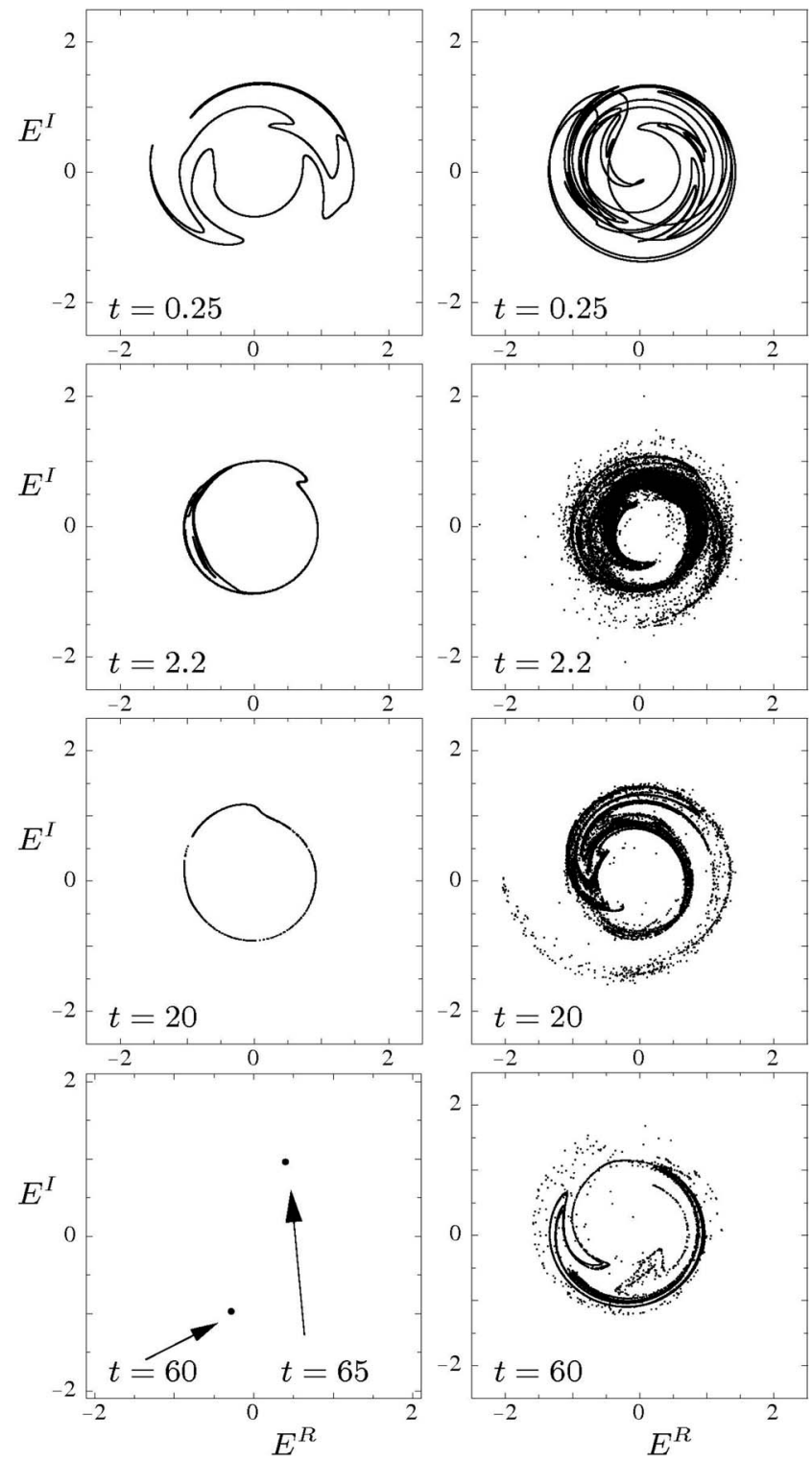

FIG. 6. The two different types of dynamics found in a single laser with external noise are shown as snapshots of 50000 trajectories at different times $t$ in the projection onto the complex $E$ plane. The left column $\left(D_{\text {ext }}=0.1\right)$ shows convergence to a random sink and the right column $\left(D_{\text {ext }}=0.5\right)$ shows convergence to a random strange attractor; $\Lambda=1$ and $\alpha=3$. At $t=0$, the initial points are evenly distributed on the ellipse $\left(E^{R} / 2\right)^{2}+\left(2 E^{I}\right)^{2}=1, N=0$.

system has a random sink for $D_{\text {ext }}>0$ and $\Lambda>0$ [Fig. 5(a)]. However, for $\alpha=3$ one finds two curves of stochastic $d$ bifurcation where the largest LE crosses through zero [Fig. 5(b)]. For parameter settings between these two curves the system has a random strange attractor and the synchronous solution (7) is unstable. Hence, the synchronizationdesynchronization transitions of $M$ uncoupled lasers with common external noise are caused by the stochastic $d$ bifurcation to the random strange attractor in a single laser with external noise. Examples of two qualitatively different dynamics found for $\alpha=3$ are shown in Fig. 6 as snapshots of 50000 trajectories at different times $t$. In column 6(a),
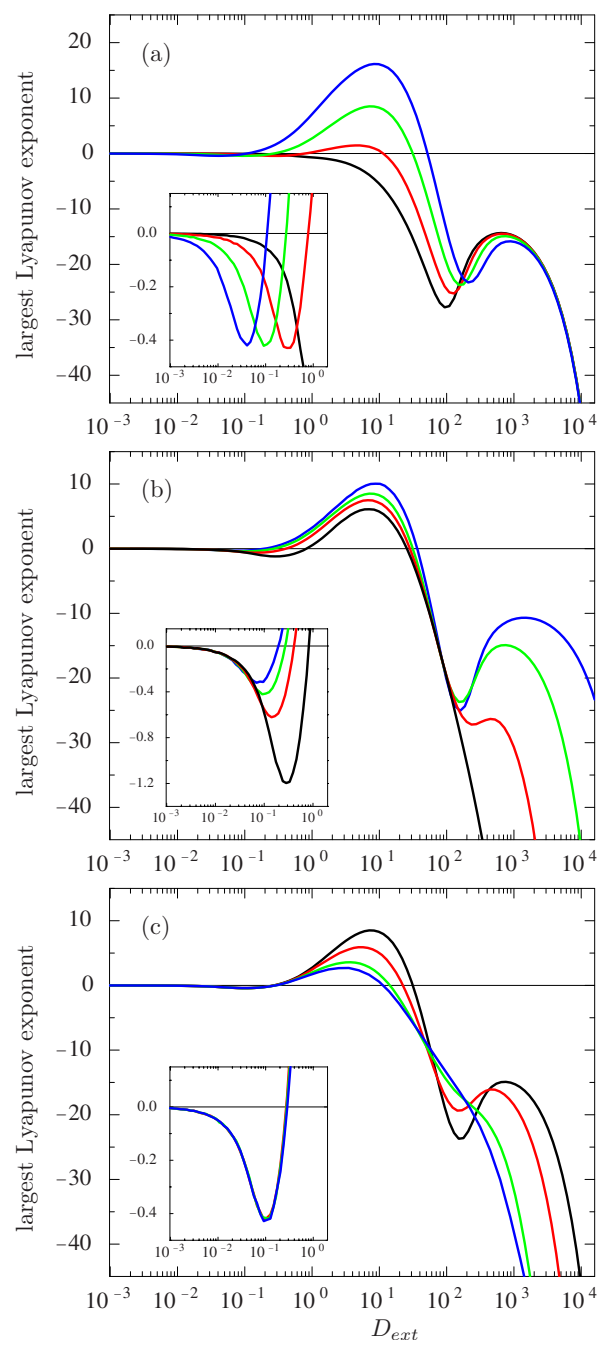

FIG. 7. (Color online) The largest Lyapunov exponent vs $D_{\text {ext }}$ in a single laser with $\Lambda=1$. In panel (a) $\beta=2.77, \gamma=500$ and from top to bottom $\alpha=$ (blue) 5.0, (green) 3.0, (red) 1.5, (black) 0.0. In panel (b) $\alpha=3$ and from top to bottom the cavity decay rate=(blue) 2 $\times 10^{12} \mathrm{~s}^{-1}(\beta=1.88, \gamma=1000)$, (green) $10^{12} \mathrm{~s}^{-1}(\beta=2.77, \gamma=500)$, (red) $5 \times 10^{11} \mathrm{~s}^{-1}(\beta=4.53, \gamma=250)$, (black) $2 \times 10^{11} \mathrm{~s}^{-1}(\beta=9.82$, $\gamma=100$ ). In panel (c) $\beta=2.77, \alpha=3$ and from top to bottom the carrier decay rate $=($ black $) 10^{9} \mathrm{~s}^{-1} \quad(\gamma=500), \quad($ red $) 2 \times 10^{9} \mathrm{~s}^{-1}$ $(\gamma=250), \quad($ green $) 5 \times 10^{9} \mathrm{~s}^{-1} \quad(\gamma=100), \quad$ (blue) $8 \times 10^{9} \mathrm{~s}^{-1}$ $(\gamma=62.5)$. The insets are expanded plots showing the first minimum.

nearby trajectories representing independent lasers converge to the random sink which itself moves randomly in time. In column 6(b), the trajectories converge to the random strange attractor on which they move continuously apart so giving rise to the fractal-like structure that evolves in time.

To establish persistence of the stochastic $d$ bifurcation in various laser designs we studied the largest LE in dependence on $\alpha$ and two other laser parameters, namely, photon and carrier decay rates. Typically, a curve of the largest LE plotted versus $D_{e x t}$ in Fig. 7 has two local minima and maxima. The first maximum has positive largest LE across a wide range of photon [Fig. 7(b)] and carrier [Fig. 7(c)] decay rates provided that $\alpha$ is large enough [Fig. 7(a)]. Therefore, bifurcation to random strange attractor is expected in most 


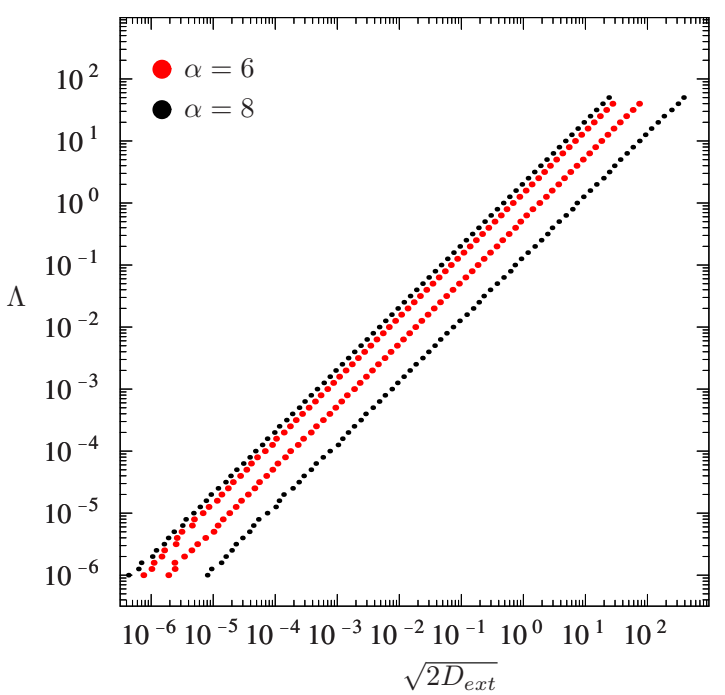

FIG. 8. (Color online) The two branches of the stochastic $d$ bifurcation in the Hopf normal form with external noise, Eq. (8), plotted in the $\left(\sqrt{2 D_{\text {ext }}}, \Lambda\right)$ plane for (two inner lines, red) $\alpha=6$ and (two outer lines, black) $\alpha=8$. See Fig. 10 for comparison with the laser model. Each dot was calculated numerically by detecting a change in the sign of the largest Lyapunow exponent from negative (positive) to positive (negative) for the left (right) branch.

single-mode semiconductor lasers with external optical noise. Moreover, we found that a random strange attractor can also be induced by external carrier noise alone (not shown), represented by an additional $\delta$-correlated random Gaussian process on the right-hand side of Eq. (2).

\section{B. Comparison with Hopf normal form}

The stochastic $d$ bifurcation uncovered in the previous section has been reported only in biological systems $[7-9,12,13]$, but it should also appear in other noise-driven limit-cycle oscillators. Here, we use the laser model in conjunction with the Hopf normal form to contribute to a better understanding of this phenomenon. Specifically, we address its dependence on the three parameters: $D_{e x t}, \Lambda$, and $\alpha$. With an exception of certain approximations [9], this general problem is beyond the reach of analytical techniques and so numerical analysis is the tool of choice.

To identify effects characteristic to our particular laser model, we first consider the normal form of a Hopf bifurcation with amplitude-phase coupling and additive white Gaussian noise [30]

$$
\frac{d z}{d t}=\left\{\Lambda+i\left[1-\alpha\left(\Lambda-|z|^{2}\right)\right]\right\} z-z|z|^{2}+f_{\text {ext }}(t),
$$

where $z \in \mathrm{C}$ and $f_{\text {ext }}(t)$ is defined by Eqs. (6). If $f_{\text {ext }}(t)=0$, the system has an equilibrium for all values of $\Lambda$. This equilibrium is stable if $\Lambda<0$ and unstable if $\Lambda>0$. Moreover, there is a stable limit cycle if $\Lambda>0$ that is born via Hopf bifurcation at $\Lambda=0$. The (higher-order) term $i \alpha\left(\Lambda-|z|^{2}\right) z$ representing amplitude-phase coupling does not affect topological properties of Eq. (8) if $f_{\text {ext }}(t)=0$. Therefore, this term does not usually appear in the Hopf normal form. However, if

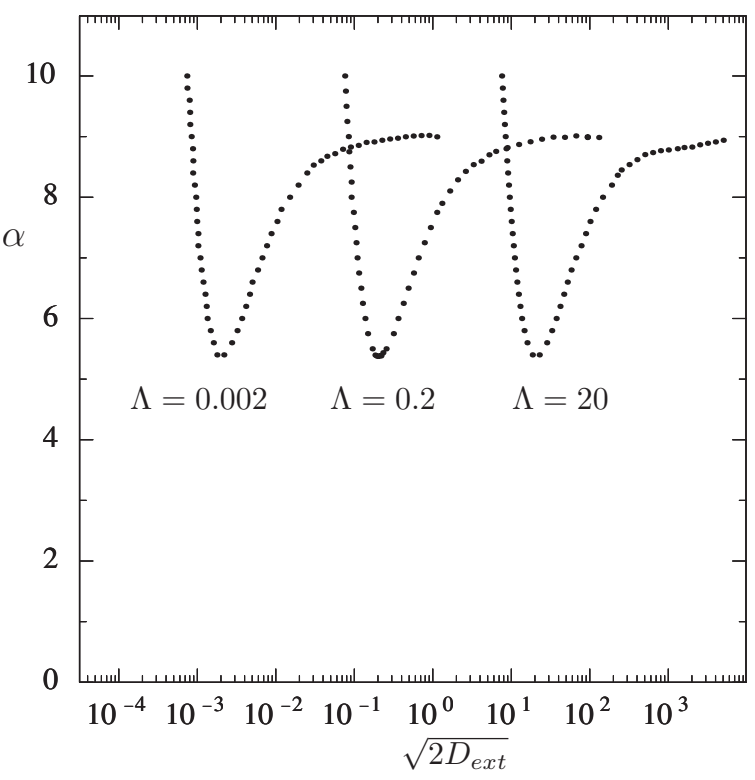

FIG. 9. The stochastic $d$ bifurcation in the Hopf normal form with external noise, Eq. (8), plotted in the $\left(\sqrt{2 D_{\text {ext }}}, \alpha\right)$ plane for $\Lambda$ $=0.002, \Lambda=0.2$, and $\Lambda=20$. See Fig. 11 for comparison with the laser model. Each dot was calculated as in Fig. 8.

$f_{\text {ext }}(t) \neq 0$, this term has to be included since its omission would give a system that is not topologically equivalent to Eq. (8).

Figures 8 and 9 show the dependence of the noiseinduced $d$ bifurcation on $\Lambda, D_{e x t}$, and $\alpha$ in Eq. (8). In the three-dimensional parameter space, the two-dimensional surface of $d$ bifurcation has a ridge at $\alpha_{\min } \approx 5.3$ and appears to be asymptotic to $\alpha \approx 9$ with increasing $D_{\text {ext }}$ (Fig. 9). Furthermore, numerical results in Fig. 9 suggest that the shape of the $d$-bifurcation curve in the two-dimensional section $\left(D_{e x t}, \alpha\right)$ is independent of $\Lambda$. As a consequence, for fixed $\alpha$ within the range $5.3 \lesssim \alpha \lesssim 9$ one finds two $d$-bifurcation curves (Fig. 8) parametrized by

$$
\Lambda=C_{1,2}(\alpha) \sqrt{2 D_{\text {ext }}},
$$

and bounding the region of noise-induced chaos in the $\left(D_{\text {ext }}, \Lambda\right)$ plane. Since $C_{1}\left(\alpha_{\text {min }}\right)=C_{2}\left(\alpha_{\text {min }}\right)=1$, these two curves merge into a single curve

$$
\Lambda=\sqrt{2 D_{e x t}}
$$

and the chaotic region disappears from the $\left(D_{\text {ext }}, \Lambda\right)$ plane when $\alpha=\alpha_{\text {min }}$. For $\alpha \geq 9$, one finds only one bifurcation curve in the $\left(D_{\text {ext }}, \Lambda\right)$ plane, hence the chaotic region remains unbounded on the right-hand side (Fig. 9). Finally, the stochastic $d$ bifurcation appears to originate from the half line $\left(D_{\text {ext }}=0, \quad \Lambda=0, \quad \alpha \gtrsim 5.3\right)$ of the deterministic Hopf bifurcation.

Similar results are expected for any noise-driven Hopf oscillator with amplitude-phase coupling, at least in the limit of small $\Lambda$. This is because near to Hopf bifurcation such systems can be reduced to Eq. (8) using center manifold theory [30]. In particular, the deterministic part of Eqs. (1) and (2) can be reduced to 


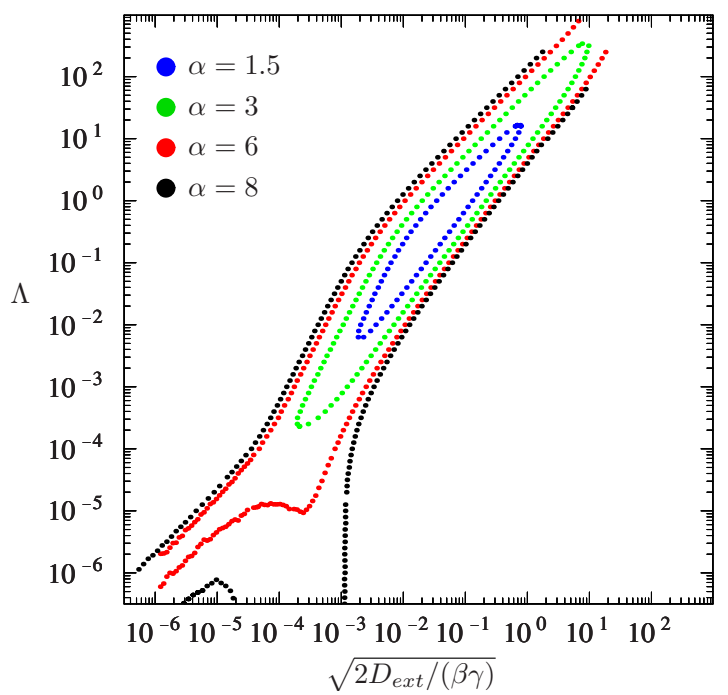

FIG. 10. (Color online) The stochastic $d$ bifurcation in laser with no spontaneous emission noise and with external noise [equations (1) and (2)] plotted in the $\left[\sqrt{2 D_{\text {ext }} /(\beta \gamma)}, \Lambda\right]$ plane. From inner to outer contour $\alpha=$ (blue) 1.5, (green) 3, (red) 6, and (black) 8. See Fig. 8 for comparison with the Hopf normal form. Each dot was calculated as in Fig. 8.

$$
\frac{1}{\beta \gamma} \frac{d E}{d t}=\left[\Lambda+i\left(\frac{\Delta}{\beta \gamma}-\alpha\left(\Lambda-|E|^{2}\right)\right)\right] E-E|E|^{2}
$$

on the two-dimensional invariant center manifold

$$
W^{c}=\left\{(E, N): N=\Lambda-|E|^{2}\right\} .
$$

After rescaling time $\tilde{t}=t \beta \gamma$ and detuning $\tilde{\Delta}=\Delta /(\beta \gamma)$, one obtains an approximated model of a single laser with external signal

$$
\frac{d E}{d \widetilde{t}}=\left\{\Lambda+i\left[\widetilde{\Delta}-\alpha\left(\Lambda-|E|^{2}\right)\right]\right\} E-E|E|^{2}+f_{e x t}(\widetilde{t}),
$$

that is identical to Eq. (8) if $\widetilde{\Delta}=1$. For the rescaled time $\widetilde{t}$, the external-signal correlations become

$$
\left\langle f_{\text {ext }}^{R}(\widetilde{t}) f_{\text {ext }}^{R}\left(\widetilde{t}^{\prime}\right)\right\rangle=\left\langle f_{\text {ext }}^{I}(\widetilde{t}) f_{\text {ext }}^{I}\left(\widetilde{t}^{\prime}\right)\right\rangle=\frac{D_{\text {ext }}}{\beta \gamma} \delta\left(\widetilde{t}-\widetilde{t}^{\prime}\right) .
$$

These theoretical considerations are supported with numerical calculations in Figs. 10 and 11. For a fixed $\alpha$, Eq. (8) and Eqs. (1) and (2) give identical results if the external noise is weak enough but significant discrepancies arise with increasing noise strength. First of all, it is possible to have onedimensional sections for fixed $\Lambda$, as those in Fig. 7, with two uplifts of the largest LE to positive values [black dots for $\Lambda<10^{-6}$ in Fig. 10]. Second, the chaotic region in Eqs. (1) and (2) expands toward much lower values of $\alpha$. Third, in the full laser model the shape of the two-dimensional surface of $d$ bifurcation becomes strongly dependent on $\Lambda$ (distorted) and has a minimum rather than a ridge. As a consequence, although the stochastic $d$ bifurcation seems to originate from the half line $\left(D_{\text {ext }}=0, \Lambda=0, \alpha \gtrsim 5.3\right)$ of the deterministic Hopf bifurcation, it will appear only as a closed and isolated curve away from the origin of the $\left(D_{\text {ext }}, \Lambda\right)$ plane if $1 \lesssim \alpha$

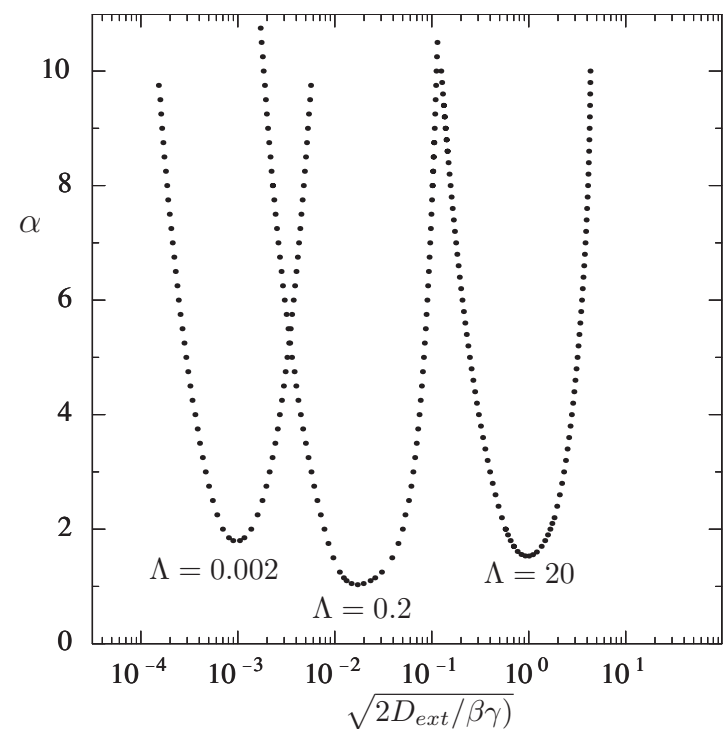

FIG. 11. The stochastic $d$ bifurcation in laser with no spontaneous emission noise and with external noise [equations (1) and (2)] plotted in the $\left[\sqrt{2 D_{\text {ext }} /(\beta \gamma)}, \alpha\right]$ plane for $\Lambda=0.002, \Lambda=0.2$, and $\Lambda$ $=20$. Each dot was calculated as in Fig. 8

$\$ 5.3$ (Fig. 10). Finally, the laser chaotic region remains bounded within a finite range of $D_{\text {ext }}$ even for large $\alpha$.

The onset of discrepancies between Eq. (8) and Eqs. (1) and (2) coincides with changes in transient dynamics toward the limit cycle (the "on" state) of the noise-free laser. Because the laser limit cycle is an $\mathrm{S}^{1}$ group orbit, it can be transformed to a circle of nonhyperbolic (neutrally stable) equilibria by setting $\Delta=0$ in Eq. (1). Floquet exponents of the laser limit cycle are then calculated analytically as eigenvalues of one of the (infinitely many) equilibria. Specifically, if $0<\Lambda<\{4 \gamma[1-\sqrt{1-1 /(2 \gamma)}]-1\} \beta^{-1} \approx 9 \times 10^{-5}$, the overdamped limit cycle has three real Floquet exponents

$$
\mu_{1}=0, \quad \mu_{2,3}=-a \pm b,
$$

and if $\Lambda>\{4 \gamma[1-\sqrt{1-1 /(2 \gamma)}]-1\} \beta^{-1} \approx 9 \times 10^{-5}$ the underdamped limit cycle has one real and two complex-conjugate Floquet exponents

$$
\mu_{1}=0, \quad \mu_{2,3}=-a \pm i b,
$$

where $a=-\frac{1}{2}(1+\beta \Lambda)>0$ and $b=\sqrt{\left|a^{2}-2 \beta \gamma \Lambda\right|}>0$. In the laser literature, the oscillatory relaxation found for $\Lambda$ $\gtrsim 9 \times 10^{-5}$ is called relaxation oscillation. (Note that in dynamical systems the term relaxation oscillation is usually used to describe a completely different phenomenon of selfsustained oscillations of slow-fast nature.)

To unveil the link between the transient dynamics and the stochastic bifurcation, we plot $\Lambda$ versus the real parts of the nonzero Floquet exponents or LEs in Fig. 12; note that Floquet exponents and LEs are related by $\mathrm{LE}_{i}=\operatorname{Re}\left[\mu_{i}\right]$. A comparison between Figs. 8, 10, and 12 shows strong correlation between the type and rate of relaxation toward the limit cycle in the noise-free system and the shape of the noise-induced $d$ bifurcation. In the deterministic Hopf normal form (8), the linear relation $\Lambda=-\mu_{2} / 2$, between $\Lambda$ and the nonzero Flo- 


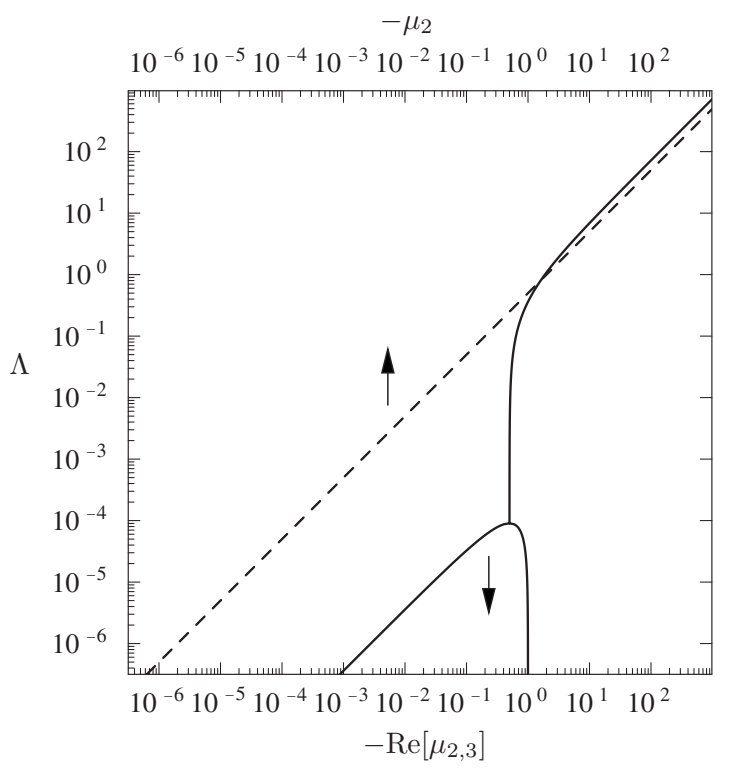

FIG. 12. $\Lambda$ versus (solid) the two nonzero LEs of the deterministic laser system (1) and (2) and (dashed) the nonzero LE of the deterministic Hopf normal form (8).

quet exponent $\mu_{2}$ (dashed line in Fig. 12), results in a linear parametrization (9) of the $d$ bifurcation curves in the $\left(\Lambda, \sqrt{2 D_{\text {ext }}}\right)$ plane (Fig. 8). In the deterministic laser system (1) and (2), the nonlinear relation (12) and (13) between $\Lambda$ and the nonzero Floquet exponents $\mu_{2,3}$ (solid curves in Fig. 12) results in a very similar nonlinear parametrization of the $d$-bifurcation curves in the $\left(\Lambda, \sqrt{2 D_{e x t}}\right)$ plane (Fig. 10). The splitting up of the chaotic region bounded by the black dots at low $\Lambda$ (Fig. 10) is related to two different time scales of transient dynamics along the two eigendirections orthogonal to the limit cycle (the corresponding two real Floquet multipliers are shown by the solid curve in Fig. 12). Finally, the appearance of oscillatory relaxation in the laser system is associated with a significant expansion of the chaotic region toward small $\alpha$.

We remark that this section neglected the intrinsic noise to numerically study some universal features of the stochastic $d$ bifurcation. In a real semiconductor-laser experiment one has to deal with the intrinsic spontaneous emission noise which is typically comparable to $\sqrt{D_{e x t} /(\beta \gamma)} \leqq 0.0045$.

\section{NOISE-INDUCED STRANGE ATTRACTORS}

Complicated invariant sets, such as strange attractors, require a balanced interplay between phase-space expansion and contraction [30]. If phase-space expansion in certain directions is properly compensated by phase-space contraction in some other directions, nearby trajectories can separate exponentially fast (positive LE) and yet remain within a finite subset of the (infinite) phase space. Figure 13 uses Eqs. (1) and (2) to illustrate how the interplay between phase-space expansion and contraction near a stable limit cycle can produce fold-and-stretch action which is responsible for the formation of a strange attractor. If $\Lambda=1$, the stable limit cycle is given by $\left(\left|E_{0}\right|^{2}, N_{0}\right)=(1,0)$. At time $t=0$, the entire cycle is
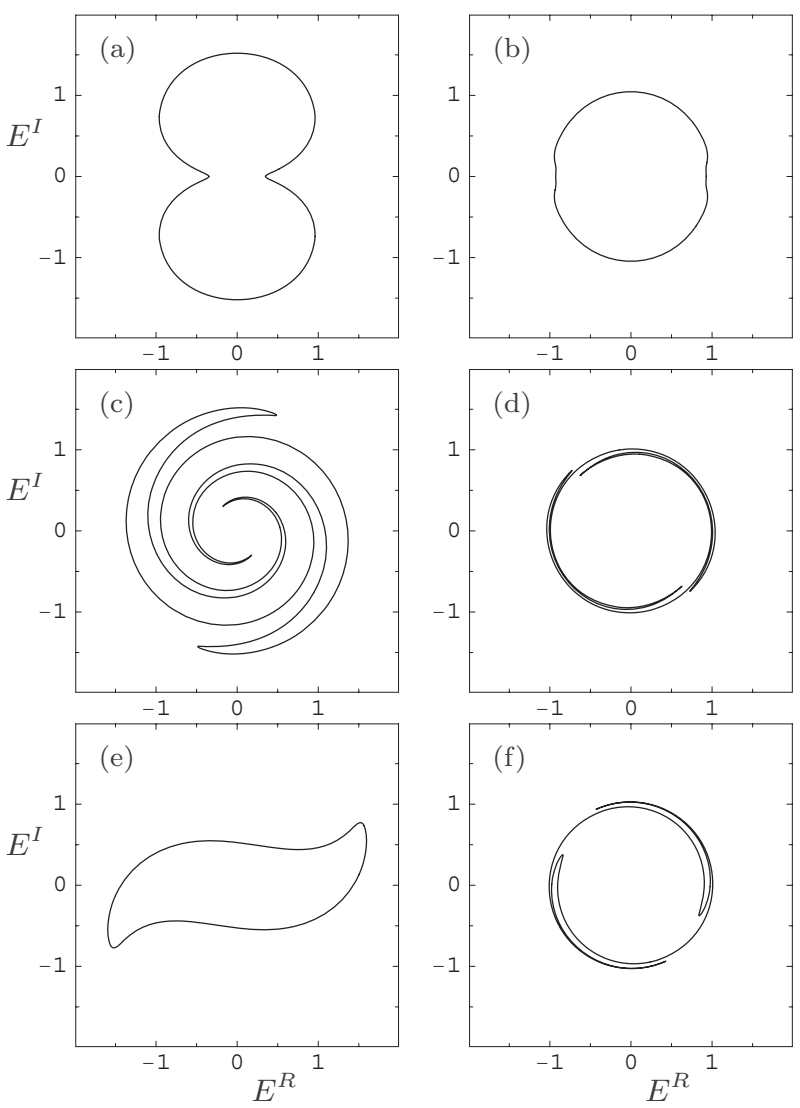

FIG. 13. Projection onto the complex $E$ plane with snapshots of 5000 trajectories at times [panels (a), (c), (e)] $t=\tilde{t}=0.06$ and [panels (b), (d), (f)] $t=\tilde{t}=1.28$ for [panels (a), (b)] Eqs. (1) and (2) with $\alpha$ $=0$, [panels (c), (d)] Eqs. (1) and (2) with $\alpha=3$, and [panels (e), (f)] Eq. (11) with $\alpha=3 . \Lambda=1$ and at $t=0$ the initial points are evenly distributed on the ellipse $\left(E^{R} / 2\right)^{2}+\left(2 E^{I}\right)^{2}=1, N=0$.

perturbed to the shape of an ellipse with $\left(E^{R} / P\right)^{2}+\left(P E^{I}\right)^{2}$ $=1, N=0$; the perturbation has to be nonconstant along the cycle ( $\mathrm{S}^{1}$ breaking). If $\alpha=0$, the perturbed set always relaxes back to the cycle with no stretching or folding [Figs. 13(a) and 13(b)]. However, if $\alpha>0$, the phase-amplitude coupling makes the perturbed points with $|E|>1$ rotate faster than the points with $|E|<1$, hence, giving rise to stretching along the cycle [Figs. 13(c) and 13(e)]. In particular, for any $|\alpha|>0$ there is a sufficiently large perturbation $P$ such that the interplay between stretching along the cycle and concurrent attraction toward the cycle produces noticeable folds [Figs. 13(d) and 13(f)]. Repeating the perturbation having nonconstant strength along the cycle at periodic or random time intervals can give rise to strange attractors [13]. A rigorous proof of strange attractor formation in periodically perturbed limit-cycle oscillators with amplitude-phase coupling is given in Ref. [31].

In contrast to discrete-time perturbation [31], white noise is a continuous-time perturbation and may have different effects. First of all, we demonstrated that purely additive noise is sufficient to induce random strange attractors. Furthermore, numerical analysis in Sec. IV B shows that noiseinduced strange attractors require a different balance between the amount of amplitude-phase coupling, rate of 


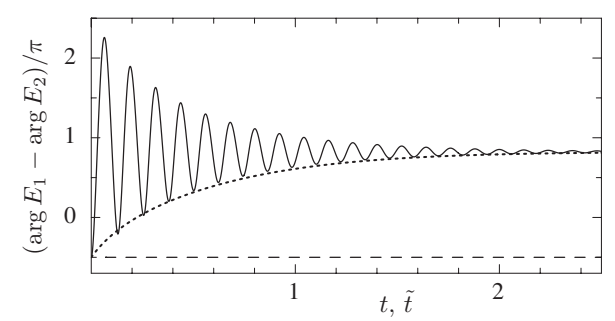

FIG. 14. Phase-space stretching along the limit cycle shown as a change in the phase difference between two trajectories starting at $\left(E_{1}^{R}, E_{1}^{I}, N_{1}\right)=(2,0,0)$ and $\left(E_{2}^{R}, E_{2}^{I}, N_{2}\right)=(0,0.5,0)$, respectively. The three curves are obtained using (dashed) Eqs. (1) and (2) with $\alpha=0$, (solid) Eqs. (1) and (2) with $\alpha=3$, and (dotted) Eq. (11) with $\alpha=3$.

relaxation toward the limit cycle, and strength of external perturbation. Unlike in the case of discrete-time perturbation, amplitude-phase coupling has to be strong enough $(|\alpha|$ $\left.>\left|\alpha_{\text {min }}\right|>0\right)$ to allow sufficient stretch-and-fold action and $\left|\alpha_{\min }\right|$ does not depend on the external-noise strength. Furthermore, provided that the amplitude-phase coupling is strong enough, the external-noise strength needs to be at least comparable to the relaxation rate toward the limit cycle to allow formation of random strange attractors. Often, noise-induced strange attractor exists within a finite interval of the external-noise strength bounded by two stochastic $d$ bifurcations (see also Refs. [8,9]). Finally, different relaxation rates toward the limit cycle associated with different eigendirections may give rise to more than one parameter interval with noise-induced strange attractor.

The above considerations help to explain intuitively findings in Sec. IV B, in particular, the chaotic region expansion associated with a change in the type of relaxation toward the limit cycle. For $\Lambda \gtrsim 10^{-1}$, Eqs. (1) and (2) and Eq. (11) have nearly identical relaxation time scales toward the limit cycle (Fig. 12). However, despite identical amplitude-phase coupling $\alpha$, they show different dynamics along the limit cycle. Owing to one additional degree of freedom and oscillatory relaxation (13) in the laser system, the instantaneous stretching along the limit cycle in the vector field (1) and (2) can be much stronger compared to the vector field (11), especially at short times after the perturbation. This effect is illustrated by the time evolution of the phase difference between two trajectories starting at different isochrones for $\alpha=3$ in Fig. 14 . In both vector fields, the phase difference approaches the same limit value at large $t$. However, at small $t$, the oscillatory phase difference of Eqs. (1) and (2) exceeds significantly the monotonically varying phase difference of Eq. (11) [compare Figs. 13(c) and 13(e) as well as solid and dotted curves in Fig. 14]. We conjecture that the enhancement in the stretch-and-fold action arising from laser's relaxation oscillation results in a smaller $\alpha_{\min }$ required to induce chaos for given $\Lambda$.

\section{CONCLUSIONS}

This paper uses semiconductor-laser rate equations in conjunction with normal form of Hopf bifurcation to study noise-induced synchronization and chaos in limit-cycle oscillators with amplitude-phase coupling.

While external white optical noise can induce synchrony in an ensemble of uncoupled and intrinsically noisy lasers, the parameter region with synchronous dynamics becomes interrupted with an interval(s) of asynchronous dynamics if the amplitude-phase coupling is sufficiently large. Stability analysis shows that synchronous solution of the whole ensemble loses stability via stochastic $d$ bifurcation to a random strange attractor in a single noise-driven laser. We performed systematic study of this bifurcation with dependence on the three parameters: the Hopf bifurcation parameter (laser pump), the amount of amplitude-phase coupling (linewidth enhancement factor), and the external-noise strength. In this way, we uncovered a vast parameter region with purely noise-induced chaos not observed in optical systems to date. Furthermore, we contributed to the better understanding of synchronization-desynchronization transition in noise-driven oscillators.

More specifically, in the three-dimensional parameter space, the two-dimensional surface of the stochastic bifurcation originates from the half line of the deterministic Hopf bifurcation. In the plane of external-noise strength and Hopf parameter, one finds stochastic bifurcation curve(s) if the amplitude-phase coupling is strong enough. The shape of these noise-induced bifurcation curves is determined by the type and rate of the relaxation toward the limit cycle in the noise-free system. As a consequence of center manifold theory, at low external noise the stochastic bifurcation curve(s) are expected to follow numerically observed squareroot law (9) in all noise-driven Hopf oscillators. However, for high external noise one expects deviations from this law. Deviations arise because different systems experience different effects of higher-order terms and additional degrees of freedom on the relaxation toward the cycle. In the laser example, the parameter region with random strange attractor is in perfect agreement with the result obtained from the Hopf normal form provided that the external noise is sufficiently low. However, the laser's chaotic region becomes significantly distorted as the external noise increases: it splits up at lower external noise and expands toward smaller amplitudephase coupling at higher external noise. We intuitively explained this result by demonstrating how the appearance of damped relaxation oscillation toward the laser limit cycle can greatly increase phase-space stretching and folding which are necessary for creation of a chaotic attractor.

\section{ACKNOWLEDGMENTS}

I would like to thank P. Ashwin, M. Holland, and S. Townley for useful comments. 
[1] A. S. Pikovsky, in Nonlinear and Turbulent Processes in Physics, edited by R. Z. Sagdeev (Harwood Academic, Singapore, 1984), pp. 1601-1604.

[2] Z. F. Mainen and T. J. Sejnowski, Science 268, 1503 (1995).

[3] R. V. Jensen, Phys. Rev. E 58, R6907 (1998).

[4] C. S. Zhou, J. Kurths, E. Allaria, S. Boccaletti, R. Meucci, and F. T. Arecchi, Phys. Rev. E 67, 066220 (2003).

[5] J. N. Teramae and D. Tanaka, Phys. Rev. Lett. 93, 204103 (2004).

[6] A. Uchida, R. McAllister, and R. Roy, Phys. Rev. Lett. 93, 244102 (2004).

[7] E. Kosmidis and K. Pakdaman, J. Comput. Neurosci. 14, 5 (2003).

[8] D. S. Goldobin and A. Pikovsky, Phys. Rev. E 71, 045201(R) (2005).

[9] D. S. Goldobin and A. Pikovsky, Phys. Rev. E 73, 061906 (2006).

[10] N. F. Rulkov, M. M. Sushchik, L. S. Tsimring, and H. D. I. Abarbanel, Phys. Rev. E 51, 980 (1995).

[11] A. S. Pikovsky, M. Rosenblum, and J. Kurths, Synchronization-A Unified Approach to Nonlinear Science (Cambridge University Press, Cambridge, UK, 2001).

[12] K. K. Lin, E. Shea-Brown, and L.-S. Young, e-print arXiv:0708.3061v2; e-print arXiv:0708.3063v2.

[13] K. K. Lin and L.-S. Young, Nonlinearity 21, 899 (2008).

[14] C. H. Henry, IEEE J. Quantum Electron. 18, 259 (1982).

[15] Typical semiconductor lasers have linewidth enhancement factors in the range between 0 and 10 .

[16] C. O. Weiss and R. Vilaseca, Dynamics of Lasers (VCH Verlagsgesellschaft, Weinheim, Germany, 1991).

[17] W. W. Chow, M. O. Scully, and E. W. van Stryland, Opt. Commun. 15, 6 (1975).

[18] Diode Laser Arrays, edited by D. Boetz and D. R. Scifres
(Cambridge University Press, Cambridge, UK, 1994).

[19] R. Lang, IEEE J. Quantum Electron. 18, 976 (1982).

[20] S. Wieczorek, B. Krauskopf, T. B. Simpson, and D. Lenstra, Phys. Rep. 416, 1 (2005).

[21] S. Wieczorek and W. W. Chow, Opt. Commun. (to be published).

[22] Alternatively, for a limit cycle of period $T$ one can define a time-shift map $\varphi_{T}: x(t) \rightarrow x(t+T)$ which fixes any point on the limit cycle. Then, an isochron is an invariant stable manifold $W^{s}(p)=\left\{x: \varphi_{T}^{n}(x) \rightarrow p, n \rightarrow \infty\right\}$.

[23] $\gamma, \beta, 2 D_{E}$, and $2 D_{N}$ are calculated as in Ref. [21] assuming realistic values of the refractive index $=3.4$, photon decay rate $=10^{12} \mathrm{~s}^{-1}$, carrier decay rate $=10^{9} \mathrm{~s}^{-1}$, differential gain $=10^{-19} \mathrm{~m}^{2}$, carrier density at transparency $=10^{24} \mathrm{~m}^{-3}$, confinement factor $=0.05$, and spontaneous emission coefficient $C_{s p}$ $=10^{-5}$.

[24] This phenomenon was studied in [17] as a thermodynamic phase transition.

[25] T. Erneux, V. Kovanis, A. Gavrielides, and P. M. Alsing, Phys. Rev. A 53, 4372 (1996).

[26] K. E. Chlouverakis and M. J. Adams, Opt. Commun. 216, 405 (2003).

[27] C. Bonatto and J. A. C. Gallas, Phys. Rev. E 75, 055204(R) (2007).

[28] S. Wieczorek, T. B. Simpson, B. Krauskopf, and D. Lenstra, Phys. Rev. E 65, 045207(R) (2002).

[29] L. Arnold, Random Dynamical Systems (Springer-Verlag, Berlin, 1998).

[30] J. Guckenheimer and P. Holmes, Nonlinear Oscillations, Dynamical Systems, and Bifurcations of Vector Fields (SpringerVerlag, New York, 1983).

[31] Q. Wang and L.-S. Young, Commun. Math. Phys. 240, 509 (2003). 